\title{
Curve shortening flow in heterogeneous media
}

\author{
Annalisa Cesaroni, Matteo Novaga \\ Dipartimento di Matematica Pura e Applicata, Università di Padova, \\ via Trieste 63, 35121 Padova, Italy \\ E-mail: acesar@math.unipd.it,novaga@math.unipd.it \\ ENRICO VALDINOCI \\ Dipartimento di Matematica, Università di Milano, \\ via Saldini 50, 20133 Milano, Italy \\ E-mail: enrico.valdinoci@unimi.it
}

[Received 5 August 2010 and in revised form 13 October 2011]

\begin{abstract}
In recent years, there has been a growing interest in geometric evolutions in heterogeneous media. Here we consider curvature driven flows of planar curves with an additional space-dependent forcing term, and we look for estimates which depend only on the $L^{\infty}$-norm of the forcing term. Our motivation comes from a homogenization problem, which we can rigorously solve in the special case when the initial curve is a graph and the forcing term does not depend on the vertical direction. In such case, we are also able to define a solution of the evolution even if the forcing term is just a bounded function, not necessarily continuous.
\end{abstract}

\section{Introduction}

In this paper we consider the curvature shortening flow of planar curves in a heterogeneous medium, which is modeled by a spatially-dependent additive forcing term. The evolution law reads

$$
v=(\kappa+g) \nu,
$$

where $v$ is the inward normal vector to the curve, $\kappa$ is the curvature of the curve, $v$ is the normal velocity vector, and $g \in L^{\infty}\left(\mathbb{R}^{2}\right)$ represents the forcing term.

The original motivation for our analysis comes from a homogenization problem related to the averaged behaviour of an interface moving by curvature plus a rapidly oscillating forcing term. More precisely, the evolution law is given by

$$
v=\left(\kappa+g\left(\frac{x}{\varepsilon}, \frac{y}{\varepsilon}\right)\right) \nu,
$$

where $g$ is a 1-periodic Lipschitz continuous function.

When the forcing term is periodic, equation (1) was recently considered in [6], where the authors prove existence and uniqueness of planar pulsating waves in every direction of propagation. This result leads to the homogenization of (2) for plane-like initial data (see Section 3). Related results on the homogenization of interfaces moving with normal velocity given by

$$
v=\left(\varepsilon \kappa+g\left(\frac{x}{\varepsilon}, \frac{y}{\varepsilon}\right)\right) v
$$

have been obtained in [5] and [12], under suitable assumptions on the forcing term including the fact that it does not change sign, and in [4] under more general assumptions. In particular, the authors 
show that the homogenized evolution law, when it exists, is a first order anisotropic geometric law of the form $v=\bar{c}(v) v$.

Coming back to our problem, as a first step we look for geometric estimates for solutions to (1), which depend only on the $L^{\infty}$-norm of $g$. In particular, reasoning as in the case of the unperturbed curvature flow $[9,2,11]$, in Section 2 we classify all possible singularities which can arise during the evolution. As a consequence, in Section 2.5 we can show that, when $g$ is smooth and the initial curve is embedded, the existence time of a regular solution to (1) is bounded below by a quantity depending only on $\|g\|_{\infty}$ and on the initial curve. Unfortunately, since we have no estimates on the curvature in terms of $\|g\|_{\infty}$, we are not able to obtain a general existence result for (1) in the nonsmooth case, i.e. when $g \in L^{\infty}$.

However, in Section 2.6 we overcome this difficulty by assuming that the initial curve is the graph of a function $u$, for instance in the vertical direction. In this case equation (1) becomes

$$
u_{t}=\frac{u_{x x}}{1+u_{x}^{2}}+g(x, u(x)) \sqrt{1+u_{x}^{2}} .
$$

In Lemma 2.18 we establish an $L^{p}$-estimate on $u_{x}$ which depends only on $\|g\|_{\infty}$. In Proposition 2.21 we consider a sequence of smooth forcings $g_{n}$ weakly converging to $g \in L^{\infty}$. Using the estimate on $u_{x}$ and the results of Section 2.5, and letting $u_{n}$ be the solution corresponding to $g_{n}$, we can pass to the limit as $n \rightarrow \infty$ and find that $u_{n} \rightarrow u \in H^{1}\left([0, T], L^{2}([0,1])\right) \cap L^{\infty}\left([0, T], H^{1}([0,1])\right)$, for some time $T>0$ depending only on $\|g\|_{\infty}$ and on the initial datum. When $g$ does not depend on $u$, we obtain a stronger estimate on $\left\|u_{t}\right\|_{\infty}$, which allows us to show that $u \in$ $W^{1, \infty}\left([0, T], L^{\infty}([0,1])\right) \cap L^{\infty}\left([0, T], W^{2, \infty}([0,1])\right)$.

As a first application, this leads to an existence and uniqueness result for solutions to (3), when $g$ is an $L^{\infty}$-function which is independent of $u$ (see Theorems 2.23 and 2.25).

The second application of our result is to the homogenization problem (2). In Section 3, under the assumptions of Theorem 2.23, that is, when the curve is a graph and $g$ is independent of the vertical direction, we can pass to the limit in (2) as $\varepsilon \rightarrow 0$, and show that the limit curve moves according to the evolution law

$$
v=\left(\kappa+\int_{[0,1]^{2}} g(x, y) \mathrm{d} x \mathrm{~d} y\right) \nu .
$$

\section{Local existence of solutions}

In this section we are concerned with the local existence for (1), under the assumption that the forcing term $g$ is smooth and bounded, i.e. $g \in C^{\infty}\left(\mathbb{R}^{2}\right) \cap L^{\infty}\left(\mathbb{R}^{2}\right)$. If we parametrize counterclockwise the evolving curve with a function $\gamma=\left(\gamma^{1}, \gamma^{2}\right):[0,1] \times[0, T] \rightarrow \mathbb{R}^{2}$, with $\gamma(0, \cdot)=\gamma(1, \cdot)$, problem (1) becomes

$$
\gamma_{t}=(\kappa+g) v=\frac{\gamma_{x x}^{\perp}}{\left|\gamma_{x}\right|^{2}}+g(\gamma) \frac{\left(-\gamma_{x}^{2}, \gamma_{x}^{1}\right)}{\left|\gamma_{x}\right|},
$$

where $\xi^{\perp}$ denotes the component of the vector $\xi$ orthogonal to $\gamma_{x}$. As usual we let $\tau, \nu, \kappa$ be respectively the unit tangent vector, the unit normal vector and the curvature of the evolving curve. Denoting by $s$ the arclength parameter of the curve, so that $\partial_{s}=\partial_{x} /\left|\gamma_{x}\right|$, by the classical FrenetSerret formulas we have

$$
\gamma_{s}=\tau, \quad \gamma_{s s}=\tau_{s}=\kappa \nu, \quad v_{s}=-\kappa \tau .
$$

We recall the following local in time existence result for (4), proved in [7, Thm. 4.1]. 
THEOREM 2.1 Let $\gamma_{0}:[0,1] \rightarrow \mathbb{R}^{2}$ be a smooth map such that $\gamma_{0}(0)=\gamma_{0}(1)$ and $\left|\gamma_{0}^{\prime}(x)\right|>0$ for all $x \in[0,1]$. Then there exist $T>0$ and a smooth solution to (4), defined on $[0,1] \times[0, T]$, such that $\gamma(x, 0)=\gamma_{0}(x)$ for all $x \in[0,1]$.

\subsection{Estimates on the curvature and its derivatives}

LEMMA 2.2 The following commutation rule holds:

$$
\partial_{t} \partial_{s}=\partial_{s} \partial_{t}+\kappa(\kappa+g) \partial_{s} .
$$

Moreover,

$$
\begin{aligned}
\tau_{t} & =(\kappa+g)_{s} \nu, \\
\nu_{t} & =-(\kappa+g)_{s} \tau, \\
\kappa_{t} & =(\kappa+g)_{s s}+\kappa^{2}(\kappa+g) .
\end{aligned}
$$

Proof. By definition of arclength, we have

$$
\partial_{s}=\frac{\partial_{x}}{\left|\gamma_{x}\right|}
$$

Therefore, from (4) and (5),

$$
\begin{aligned}
\partial_{t} \partial_{s}-\partial_{s} \partial_{t} & =-\left|\gamma_{x}\right|^{-3} \gamma_{x} \cdot \gamma_{x t} \partial_{x}=-\left|\gamma_{x}\right|^{-2} \tau \cdot \gamma_{x t} \partial_{x}=-\left|\gamma_{x}\right| \tau \cdot((\kappa+g) \nu)_{s} \partial_{x} \\
& =-\left|\gamma_{x}\right| \tau \cdot(\kappa+g) v_{s} \partial_{x}=\kappa(\kappa+g) \partial_{s},
\end{aligned}
$$

which is (6). Now, applying (6) to (4) and (5), we obtain

$$
\begin{aligned}
\tau_{t} & =\left(\gamma_{s}\right)_{t}=\left(\gamma_{t}\right)_{s}+\kappa(\kappa+g) \gamma_{s} \\
& =(\kappa+g)_{s} \nu+(\kappa+g) \nu_{s}+\kappa(\kappa+g) \tau=(\kappa+g)_{s} \nu,
\end{aligned}
$$

which is (7).

Also, since $|v|=1$,

$$
0=(v \cdot v)_{t} / 2=v \cdot v_{t}
$$

and so, from (7),

$$
\begin{aligned}
v_{t} & =\left(v_{t} \cdot v\right) v+\left(v_{t} \cdot \tau\right) \tau=\left(v_{t} \cdot \tau\right) \tau \\
& =\left((v \cdot \tau)_{t}-v \cdot \tau_{t}\right) \tau=-\left(v \cdot \tau_{t}\right) \tau=-(\kappa+g)_{s} \tau,
\end{aligned}
$$

which is (8), and

$$
\begin{aligned}
\kappa_{t} & =(\kappa \nu)_{t} \cdot v=\left(\tau_{s}\right)_{t} \cdot v=\left(\tau_{t}\right)_{s} \cdot v+\kappa(\kappa+g) \tau_{s} \cdot v \\
& =\left((\kappa+g)_{s} \nu\right)_{s} \cdot v+\kappa^{2}(\kappa+g)=(\kappa+g)_{s s}+\kappa^{2}(\kappa+g),
\end{aligned}
$$

which is (9).

Let us compute the evolution for the spatial derivaties of the curvature. We denote by $p_{j, k}\left(\partial_{s}^{\ell} \kappa, \partial_{s}^{m} g\right)$ a generic polynomial depending on the derivatives up to order $j$ of $\kappa$ and the derivatives up to order $k$ of $g$. 
LEMma 2.3 For all $j \in \mathbb{N}, j \geqslant 1$, we have

$$
\partial_{t} \partial_{s}^{j} \kappa=\left(\partial_{s}^{j} \kappa\right)_{s s}+\left((j+3) \kappa^{2}+(j+2) \kappa g\right) \partial_{s}^{j} \kappa+p_{j-1, j+2}\left(\partial_{s}^{\ell} \kappa, \partial_{s}^{m} g\right) .
$$

Proof. The proof is by induction on $j$. When $j=1$ from (6) and (9) we easily get

$$
\partial_{t} \kappa_{s}=\left(\kappa_{s}\right)_{s s}+\left(4 \kappa^{2}+3 \kappa g\right) \kappa_{s}+\left(\kappa^{2} g_{s}+g_{s s s}\right) .
$$

Assume now (10) for some $j \in \mathbb{N}$. Using (6), we compute recursively

$$
\begin{aligned}
\partial_{t} \partial_{s}^{j+1} \kappa & =\partial_{s} \partial_{t} \partial_{s}^{j} \kappa+\kappa(\kappa+g) \partial_{s}^{j+1} \kappa \\
& =\left(\partial_{s}^{j+1} \kappa\right)_{s s}+\left((j+3) \kappa^{2}+(j+2) \kappa g+\kappa(\kappa+g)\right) \partial_{s}^{j+1} \kappa+p_{j, j+3}\left(\partial_{s}^{\ell} \kappa, \partial_{s}^{m} g\right),
\end{aligned}
$$

which gives (10) for all $j$.

We recall that in [7] similar equations as (10) for the evolution of the second fondamental form and its derivatives are obtained for forced mean curvature flow of hypersurfaces in any dimension.

We now compute the evolution equation of $w:=\log \left|\gamma_{x}\right|$.

LEMMA 2.4 We have

$$
w_{t}=-\kappa(\kappa+g) .
$$

Proof. A direct computation using (4) gives

$$
w_{t}=\frac{\gamma_{x} \cdot \gamma_{x t}}{\left|\gamma_{x}\right|^{2}}=\tau \cdot\left(\partial_{s} \gamma_{t}\right)=-\kappa(\kappa+g)
$$

LEMmA 2.5 Assume that (4) admits a smooth solution on $[0, \bar{t}]$ with $\bar{t}>0$. Then

$$
\max _{[0,1] \times[0, \bar{t}]}\left(\partial_{s}^{j} \kappa\right)^{2} \leqslant C_{j}
$$

for all $j \in \mathbb{N}$, where the constants $C_{j}$ depend only on the initial curve, on $\bar{t}$, on $\max _{[0,1] \times[0, \bar{t}]} \kappa^{2}$ and on $\|g\|_{C^{j+2}}$.

Proof. Following [8], we let

$$
K_{j}(x, t):=\left(\partial_{s}^{j} \kappa(x, t)\right)^{2}, \quad M_{j}(t):=\max _{x \in[0,1]} K_{j}(x, t) .
$$

For all $\bar{x}$ such that $K_{j}(\bar{x}, t)=M_{j}(t)$ we have

$$
\partial_{s} K_{j}=0, \quad \partial_{s s} K_{j}=2\left(\left(\partial_{s}^{j} \kappa\right)_{s}^{2}+\partial_{s}^{j} \kappa\left(\partial_{s}^{j} \kappa\right)_{s s}\right) \leqslant 0
$$

and so

$$
\partial_{s}^{j} \kappa(\bar{x}, t)\left(\partial_{s}^{j} \kappa\right)_{s s}(\bar{x}, t) \leqslant 0 .
$$

Recalling (10), for a.e. $t \in[0, \bar{t}]$ we have

$$
\begin{aligned}
\dot{M}_{j}(t) & =\max _{\bar{x}: K_{j}(\bar{x}, t)=M_{j}(t)} \partial_{t} K_{j}=\max _{\bar{x}: K_{j}(\bar{x}, t)=M_{j}(t)} 2 \partial_{s}^{j} \kappa\left(\partial_{s}^{j} \kappa\right)_{t} \\
& =\max _{\bar{x}: K_{j}(\bar{x}, t)=M_{j}(t)} 2 \partial_{s}^{j} \kappa\left(\left(\partial_{s}^{j} \kappa\right)_{s s}+A_{j} \partial_{s}^{j} \kappa+B_{j}\right),
\end{aligned}
$$


where the constants $A_{j}, B_{j}$ depend on $M_{\ell}$ and $\|g\|_{C^{k}}$, with $\ell<j$ and $k \leqslant j+2$. Hence, using (12) we get

$$
\dot{M}_{j} \leqslant 2 A_{j} M_{j}+2 B_{j} .
$$

By Gronwall's Lemma it then follows that the quantities $M_{j}$ are uniformly bounded on $[0, \bar{t}]$.

Since the existence result in Theorem 2.1 is first established in the usual Hölder parabolic spaces $C^{k+\alpha, 2(k+\alpha)}([0,1] \times[0, T])$ (see [14]), if we still denote by $T$ the maximal existence time of the evolution, we see that, if $T<+\infty$, either $\left|\gamma_{x}\right|^{-1}$ or $\left|\partial_{s}^{j} \kappa\right|$ blow up as $t \rightarrow T$, for some $j \in \mathbb{N}$.

Proposition 2.6 Let $T$ be the maximal existence time of the evolution (4), and assume $T<$ $+\infty$. Then

$$
\lim _{t \rightarrow T}\left\|\kappa^{2}\right\|_{L^{\infty}}=+\infty .
$$

Proof. Assume for contradiction that $\kappa^{2}$ is uniformly bounded for all $t \in[0, T)$ and $x \in[0,1]$. Equation (11) implies that $\left|\gamma_{x}\right|$ and $1 /\left|\gamma_{x}\right|$ are also uniformly bounded on $[0,1] \times[0, T)$. But Lemma 2.5 implies that also the quantities $\left(\partial_{j} \kappa(x, t)\right)^{2}$ are uniformly bounded on $[0,1] \times[0, T)$ for all $j \in \mathbb{N}$, thus reaching a contradiction. We thus proved

$$
\limsup _{t \rightarrow T}\left\|\kappa^{2}\right\|_{L^{\infty}}=+\infty \text {. }
$$

Notice that the lim sup is indeed a full limit due to (9).

The following lemma provides a lower bound to (13).

LEMMA 2.7 Let $T$ be as above and assume $T<+\infty$. The following curvature lower bound holds:

$$
\liminf _{t \rightarrow T} \sqrt{T-t}\|\kappa\|_{L^{\infty}} \geqslant \frac{1}{\sqrt{2}} .
$$

Proof. Notice that (9) can be written as

$$
(\kappa+g)_{t}=(\kappa+g)_{s s}+(\kappa+g) \kappa^{2}+(\kappa+g) \nabla g \cdot v .
$$

Let $w:=(\kappa+g)^{2}$. Observe that, for $\varepsilon>0, \kappa^{2} \leqslant(1+\varepsilon) w+(1+1 / \varepsilon) g^{2}$. So, from (15) it follows that

$$
\begin{aligned}
w_{t} & =w_{s s}-2(\kappa+g)_{s}^{2}+2 w \kappa^{2}+2 w \nabla g \cdot v \\
& \leqslant w_{s s}+2 w\left((1+\varepsilon) w+\left(1+\frac{1}{\varepsilon}\right) g^{2}\right)+2\|\nabla g\|_{L^{\infty}} w \\
& \leqslant w_{s s}+2(1+\varepsilon) w^{2}+2\left(2+\frac{1}{\varepsilon}\right)\|g\|_{W^{1, \infty}} w .
\end{aligned}
$$

Letting $M:=\max _{x \in[0,1]}(\kappa+g)^{2}$, from (16) we get

$$
\frac{\mathrm{d}}{\mathrm{d} t}(M+C)=\dot{M} \leqslant 2(1+\varepsilon) M^{2}+2\left(2+\frac{1}{\varepsilon}\right)\|g\|_{W^{1, \infty}} M \leqslant 2(1+\varepsilon)(M+C)^{2},
$$

where $C=[(1+1 /(2 \varepsilon)) /(1+\varepsilon)]\|g\|_{W^{1, \infty}}^{2}$, so that

$$
-\frac{\mathrm{d}}{\mathrm{d} t} \frac{1}{M+C} \leqslant 2(1+\varepsilon) .
$$


Integrating on $[t, s] \subset[0, T)$ we thus obtain

$$
\frac{1}{M(t)+C}-\frac{1}{M(s)+C} \leqslant 2(1+\varepsilon)(s-t) .
$$

Letting now $s \rightarrow T$ and recalling that $M(s) \rightarrow+\infty$ by Proposition 2.6, we get

$$
\frac{1}{M(t)+C} \leqslant 2(1+\varepsilon)(T-t)
$$

that is,

$$
M(t) \geqslant \frac{1}{2(1+\varepsilon)(T-t)}-C
$$

which gives the conclusion.

From (17) and Proposition 2.6 we obtain the following estimate on the maximal existence time of the evolution.

Proposition 2.8 Let $T$ be the maximal existence time of (4). Then

$$
T \geqslant c\left(\left\|\kappa_{0}\right\|_{\left.L^{\infty},\|g\|_{W^{1, \infty}}\right)}\right.
$$

REMARK 2.9 Note that, in contrast to the case of curve shortening flow, in our case, due to the presence of the forcing term $g$, self-intersections may arise. Nevertheless if the initial curve is embedded then, thanks to Proposition 2.8 , it remains embedded in a time interval $\left[0, T^{\prime}\right]$, with $T^{\prime}>0$ depending only on the initial datum and on $\|g\|_{W^{1, \infty}}$.

We think it is an interesting problem to determine whether or not the constant $c$ in Proposition 2.8 depends only on the initial set and on the $L^{\infty}$-norm of $g$ (see for instance Section 2.5 below for a special case).

\subsection{Huisken's monotonicity formula}

In the following we derive a monotonicity formula for curvature flow with a forcing term, and apply it to the analysis of singularities.

By a standard computation, using the fact that $\gamma$ solves (4), we get the following formula.

LEMMA 2.10 Let $\tau>0$ and let $f: \mathbb{R}^{2} \times[0, \tau) \rightarrow \mathbb{R}$ be a smooth function. Then

$$
\frac{\mathrm{d}}{\mathrm{d} t} \int_{\gamma} f(\gamma(x(s), t), t) d s=\int_{\gamma}\left[f_{t}-\kappa(\kappa+g) f+(\kappa+g) \nabla f \cdot \nu\right] \mathrm{d} s .
$$

We denote by $L_{t}(\gamma)$ the length of the curve $\gamma([0,1], t)$, that is,

$$
L_{t}(\gamma):=\int_{0}^{1}\left|\gamma_{x}\right| \mathrm{d} x=\int_{\gamma} \mathrm{d} s .
$$

When no confusion can arise, we write $L(\gamma)$ instead of $L_{t}(\gamma)$.

COROLlary 2.11 Let $\gamma:[0,1] \times[0, T] \rightarrow \mathbb{R}^{2}$ be a solution to (4). Then

$$
L_{t}(\gamma) \leqslant L_{0}(\gamma) e^{\|g\|_{\infty}^{2} t / 2} \quad \forall t \in[0, T]
$$


Proof. Taking $f \equiv 1$ in (18) we have

$$
\frac{\partial}{\partial t} L_{t}(\gamma)=-\int_{\gamma} \kappa(\kappa+g) \mathrm{d} s \leqslant \int_{\gamma}\left(-\frac{\kappa^{2}}{2}+\frac{g^{2}}{2}\right) \mathrm{d} s
$$

which gives (19) by Gronwall's Lemma.

We now apply Lemma 2.10 with $f(p, t)=\frac{e^{-\left|p-p_{0}\right|^{2} / 4(T-t)}}{\sqrt{4 \pi(T-t)}}, p, p_{0} \in \mathbb{R}^{2}$, to get

$$
\begin{aligned}
\frac{\mathrm{d}}{\mathrm{d} t} \int_{\gamma} & \frac{e^{-\left|\gamma(x(s), t)-p_{0}\right|^{2} / 4(T-t)}}{\sqrt{4 \pi(T-t)}} \mathrm{d} s \\
= & -\int_{\gamma} \frac{e^{-\left|\gamma-p_{0}\right|^{2} / 4(T-t)}}{\sqrt{4 \pi(T-t)}}\left[\frac{\left|\gamma-p_{0}\right|^{2}}{4(T-t)^{2}}-\frac{1}{2(T-t)}+\kappa(\kappa+g)+(\kappa+g) \frac{\left(\gamma-p_{0}\right) \cdot \nu}{2(T-t)}\right] \mathrm{d} s \\
= & -\int_{\gamma} \frac{e^{-\left|\gamma-p_{0}\right|^{2} / 4(T-t)}}{\sqrt{4 \pi(T-t)}}\left[\frac{\gamma-p_{0}}{2(T-t)}+\left(\kappa+\frac{g}{2}\right) \nu\right]^{2} \mathrm{~d} s+\frac{1}{4} \int_{\gamma} \frac{e^{-\left|\gamma-p_{0}\right|^{2} / 4(T-t)}}{\sqrt{4 \pi(T-t)}} g^{2} \mathrm{~d} s \\
& +\int_{\gamma} \frac{e^{-\left|\gamma-p_{0}\right|^{2} / 4(T-t)}}{\sqrt{4 \pi(T-t)}}\left[\frac{1}{2(T-t)}+\kappa \frac{\left(\gamma-p_{0}\right) \cdot \nu}{2(T-t)}\right] \mathrm{d} s .
\end{aligned}
$$

Following [9, Theorem 3.1], the last term can be actually written as

$$
\int_{\gamma} \frac{e^{-\left|\gamma-p_{0}\right|^{2} / 4(T-t)}}{\sqrt{4 \pi(T-t)}}\left[\frac{1}{2(T-t)}+\kappa \frac{\left(\gamma-p_{0}\right) \cdot v}{2(T-t)}\right] \mathrm{d} s=\int_{\gamma} \frac{e^{-\left|\gamma-p_{0}\right|^{2} / 4(T-t)}}{\sqrt{4 \pi(T-t)}}\left|\frac{\left(\gamma-p_{0}\right) \cdot \tau}{2(T-t)}\right|^{2} \mathrm{~d} s .
$$

Substituting this in (21) we obtain an analog of Huisken's monotonicity formula ([9])

$$
\begin{aligned}
& \frac{\mathrm{d}}{\mathrm{d} t} \int_{\gamma} \frac{e^{-\left|\gamma(x(s), t)-p_{0}\right|^{2} / 4(T-t)}}{\sqrt{4 \pi(T-t)}} \mathrm{d} s \\
& \quad=\int_{\gamma} \frac{e^{-\left|\gamma(x(s), t)-p_{0}\right|^{2} / 4(T-t)}}{\sqrt{4 \pi(T-t)}}\left(-\left[\kappa+\frac{\left(\gamma(x(s), t)-p_{0}\right) \cdot v}{2(T-t)}+\frac{g}{2}\right]^{2}+\frac{1}{4} g^{2}\right) \mathrm{d} s .
\end{aligned}
$$

In the next subsection we will apply this formula in the analysis of type I singularities.

\subsection{Type I singularities}

We assume that at time $T$ the flow develops a singularity of type I, i.e. there exists a constant $C_{0}>1$ such that

$$
\max _{x \in[0,1]}|\kappa(x, t)| \leqslant \frac{C_{0}}{\sqrt{2(T-t)}} .
$$

Observe that for every $x$ and $0 \leqslant t \leqslant r<T$,

$$
|\gamma(x, r)-\gamma(x, t)| \leqslant C_{0} \sqrt{2(T-t)}-C_{0} \sqrt{2(T-r)}+\|g\|_{\infty}(r-t) .
$$


This implies that the functions $\gamma(\cdot, t)$ converge uniformly to a function $\gamma_{T}$ as $t \rightarrow T$. Now we fix $x \in[0,1]$ such that $\gamma(x, t) \rightarrow \gamma_{T}(x)=: \widehat{p}$ and $\kappa(x, t)$ becomes unbounded as $t \rightarrow T$. We rescale the curve around the point $\widehat{p}$ as follows:

$$
\tilde{\gamma}(x, z):=\frac{\gamma(x, t(z))-\widehat{p}}{\sqrt{2(T-t(z))}}, \quad z(t):=-\log \sqrt{T-t} .
$$

From (24), we deduce

$$
|\tilde{\gamma}(x, z)|=\left|\frac{\gamma(x, t(z))-\hat{p}}{\sqrt{2(T-t(z))}}\right| \leqslant C_{0}+\frac{1}{\sqrt{2}}\|g\|_{\infty} e^{-z} \leqslant C_{0}+\frac{1}{\sqrt{2}}\|g\|_{\infty},
$$

so in particular $\tilde{\gamma}(x, z)$ remains bounded as $z \rightarrow+\infty$. The evolution law satisfied by the rescaled curve $\tilde{\gamma}$ is

$$
\tilde{\gamma}_{z}=\left(\tilde{\kappa}+\sqrt{2} e^{-z} g\right) \tilde{v}+\tilde{\gamma}, \quad z \in[-\log \sqrt{T},+\infty) .
$$

We also have the rescaled version of the monotonicity formula (22): letting $y=(x-\widehat{p}) / \sqrt{2(T-t)}$, we compute

$$
\begin{aligned}
\frac{\mathrm{d}}{\mathrm{d} z} \int_{\tilde{\gamma}} e^{-|\tilde{\gamma}(x(\tilde{s}), z)|^{2} / 2} \mathrm{~d} \tilde{s}=2(T-t) \frac{\mathrm{d}}{\mathrm{d} t} \int_{\gamma} \frac{e^{-|\gamma(x(s), t)-\widehat{p}|^{2} / 4(T-t)}}{\sqrt{2(T-t)}} \mathrm{d} s \\
=\int_{\tilde{\gamma}} e^{-|\tilde{\gamma}(x(\tilde{s}), z)|^{2} / 2}\left(-\left[\tilde{\kappa}+\tilde{\gamma}(x(\tilde{s}), z) \cdot \tilde{v}+e^{-z} \frac{g}{\sqrt{2}}\right]^{2}+\frac{e^{-2 z}}{2} g^{2}\right) \mathrm{d} \tilde{s}
\end{aligned}
$$

Let $F(z):=\int_{\tilde{\gamma}} e^{-|\tilde{\gamma}(x(\tilde{s}), z)|^{2} / 2} \mathrm{~d} \tilde{s}$. Then (26) gives

$$
\frac{\mathrm{d}}{\mathrm{d} z} F(z) \leqslant \frac{\|g\|_{\infty}^{2}}{2} e^{-2 z} F(z) \leqslant \frac{\|g\|_{\infty}^{2}}{2} e^{-2 z}
$$

Integrating (27) we obtain

$$
F(z) \leqslant e^{\|g\|_{\infty}^{2} T / 4} F(-\log \sqrt{T}) \quad \forall z \geqslant-\log \sqrt{T} .
$$

In particular, we deduce that for every $R>0$ there exists a uniform bound on $\mathcal{H}^{1}(\tilde{\gamma}([0,1], z) \cap$ $B(0, R)$ ), where $\mathcal{H}^{1}$ denotes the 1-dimensional Hausdorff measure. Indeed

$$
\begin{aligned}
\mathcal{H}^{1}(\tilde{\gamma}([0,1], z) \cap B(0, R)) & =\int_{\tilde{\gamma}} \chi_{B(0, R)} \mathrm{d} \tilde{s} \leqslant \int_{\tilde{\gamma}} \chi_{B(0, R)} e^{\left(R^{2}-|\tilde{\gamma}(x, z)|^{2}\right) / 2} \mathrm{~d} \tilde{s} \\
& \leqslant e^{|R|^{2} / 2} F(z) \leqslant K
\end{aligned}
$$

for some positive constant $K$.

Proposition 2.12 Under Assumption (23), for each sequence $z_{j} \rightarrow+\infty$ there exists a subsequence $z_{j_{k}}$ such that the curve $\tilde{\gamma}\left(\cdot, z_{j_{k}}\right)$, rescaled around $\widehat{p}$, locally smoothly converges to some smooth, nonflat limit curve $\tilde{\gamma}_{\infty}$ such that

$$
\tilde{\kappa}+\tilde{\gamma}_{\infty} \cdot \tilde{v}=0
$$


Proof. The proof follows the same argument as in [9, Proposition 3.4]. Indeed, the limit curve is smooth thanks to (28), Proposition 2.6 and the fact that the rescaled curve $\tilde{\gamma}$ has uniformly bounded curvature. Moreover, it is nonflat by (14). Finally, the limit curve satisfies (29) thanks to (26) and (27).

REMARK 2.13 Proposition 2.12 implies that the type I singularities of (1) are modeled by homothetic solutions of the flow, as for the spatially homogeneous case [9]. We recall that, among such solutions, the circle is the only embedded one [1], hence, under assumption (23), $T$ is actually the extinction time for the evolution. From this we can conclude that

$$
T \geqslant c\left(\left\|\kappa_{0}\right\|_{L^{\infty}},\|g\|_{L^{\infty}}\right) .
$$

\subsection{Type II singularities}

We now consider the case that at time $T$ the flow is developing a singularity of type II, i.e.

$$
\limsup _{t \rightarrow T} \max _{x \in[0,1]}|\kappa(x, t)| \sqrt{T-t}=+\infty .
$$

PROPOSITION 2.14 Under condition (30), there exists a sequence of points and times $\left(x_{n}, t_{n}\right)$ on which the curvature blows up such that the rescaled curve along this sequence converges in $\mathcal{C}^{\infty}$ to a planar, convex limiting solution, which moves by translation.

Proof. By means of (9) and (19), an easy calculation implies that

$$
\begin{aligned}
\frac{\mathrm{d}}{\mathrm{d} t} \int_{\gamma}|\kappa| \mathrm{d} s & =-2 \sum_{x: \kappa(x, t)=0}\left|\kappa_{s}\right|+\int_{\gamma} \frac{\kappa}{|\kappa|} g_{s s} \mathrm{~d} s \\
& \leqslant\left\|\nabla^{2} g\right\|_{\infty} L_{0}(\gamma) e^{\|g\|_{\infty}^{2} t / 2}+\|\nabla g\|_{\infty} \int_{\gamma}|\kappa| \mathrm{d} s .
\end{aligned}
$$

From (31), using the Gronwall lemma, we deduce that $t \mapsto \int_{\gamma}|\kappa| \mathrm{d} s$ is uniformly bounded in $[0, T]$ and admits a bounded limit as $t \rightarrow T^{-}$.

Following [2] we choose a sequence $\left(x_{n}, t_{n}\right)$ such that

- $t_{n} \in[0, T-1 / n)$ and $t_{n}<t_{n+1}$

- $k_{n}=\left|\kappa\left(x_{n}, t_{n}\right)\right| \rightarrow+\infty$ and

$$
k_{n} \sqrt{T-1 / n-t_{n}}=\max _{t \in[0, T-1 / n]}\|\kappa\|_{\infty} \sqrt{T-1 / n-t} \rightarrow+\infty \quad \text { as } n \rightarrow+\infty .
$$

We define the new parameter $u$ as $u=k_{n}^{2}\left(t-t_{n}\right), u \in\left[-k_{n}^{2} t_{n}, k_{n}^{2}\left(T-t_{n}\right)\right]$, and the rescaled curve along the sequence $\left(x_{n}, t_{n}\right)$ as $\gamma_{n}(x, u)=k_{n}\left(\gamma(x, t(u))-\gamma\left(x_{n}, t_{n}\right)\right)$ for $x \in[0,1]$. Observe that $\gamma_{n}\left(x_{n}, 0\right)=(0,0)$ and $\kappa_{\gamma_{n}}\left(x_{n}, 0\right)=1$; moreover

$$
v_{\gamma_{n}}=\frac{\mathrm{d}}{\mathrm{d} u} \gamma_{n}=\frac{(\kappa+g(\gamma)) v}{k_{n}}=\left(\kappa_{\gamma_{n}}+g_{n}\right) v_{\gamma_{n}}
$$

where $g_{n}(y)=g\left(y / k_{n}+\gamma\left(x_{n}, t_{n}\right)\right) / k_{n}$. 
In the following, we shall write for simplicity $\kappa_{n}$ instead of $\kappa_{\gamma_{n}}$. Note that for every $\varepsilon, \omega>0$, there exists $\bar{n}$ such that $\kappa_{n}^{2} \leqslant 1+\varepsilon$ for $u \in\left[-k_{n}^{2} t_{n}, \omega\right]$. Indeed, using (32), we get

$$
\kappa_{n}^{2}(x, u)=\frac{\kappa^{2}(x, t(u))}{k_{n}^{2}} \leqslant \frac{T-1 / n-t_{n}}{T-1 / n-t(u)}=\frac{T-1 / n-t_{n}}{T-1 / n-t_{n}-u / k_{n}^{2}} .
$$

This implies that, on every bounded interval of time, the curvatures of the rescaled curves are uniformly bounded. Moreover, from this, we deduce uniform bounds also on the derivatives of the curvature, using Lemma 2.5 and recalling that $\gamma_{n}$ satisfies (33) and the fact that $\left\|\nabla^{j} g_{n}\right\|_{\infty}=$ $\left\|\nabla^{j} g\right\|_{\infty} / k_{n}^{j+1} \rightarrow 0$. By the same argument of [2, Theorem 7.3], this implies that there exists a subsequence along which the rescaled curves converge smoothly to a smooth, nontrivial limit $\gamma_{\infty}$ defined in $(-\infty,+\infty)$. Moreover $\gamma_{\infty}$ evolves by mean curvature flow, $L_{t}\left(\gamma_{\infty}\right)=+\infty$ and $\left\|\kappa_{\infty}\right\|_{\infty}=1=\left|\kappa_{\infty}(0,0)\right|$.

We prove now that $\gamma_{\infty}$ is convex. Recall that from (31) we deduce that $t \mapsto \int_{\gamma}|\kappa|$ is uniformly bounded and admits a limit as $t \rightarrow T^{-}$. The same also holds for $t \mapsto \int_{\gamma_{n}}\left|\kappa_{n}\right|$. Moreover, from (31) we also obtain

$$
\frac{\mathrm{d}}{\mathrm{d} u} \int_{\gamma_{n}}\left|\kappa_{n}\right| \mathrm{d} s=-2 \sum_{\kappa_{n}(x, u)=0}\left|\left(\kappa_{n}\right)_{s}\right|+\int_{\gamma_{n}} \frac{\kappa_{n}}{\left|\kappa_{n}\right|}\left(g_{n}\right)_{s s} \mathrm{~d} s .
$$

So

$$
\begin{aligned}
-2 \int_{-M}^{M} \sum_{\kappa_{n}=0}\left|\left(\kappa_{n}\right)_{s}\right| \mathrm{d} u & =\int_{\gamma_{n}}\left(\left|\kappa_{n}(x, M)\right|-\left|\kappa_{n}(x,-M)\right|\right) \mathrm{d} s-\int_{-M}^{M} \int_{\gamma_{n}} \frac{\kappa_{n}}{\left|\kappa_{n}\right|}\left(g_{n}\right)_{s s} \mathrm{~d} s \mathrm{~d} u \\
= & \int_{\gamma}\left(\left|\kappa\left(x, t_{n}+\frac{M}{k_{n}^{2}}\right)\right|-\left|\kappa\left(x, t_{n}-\frac{M}{k_{n}^{2}}\right)\right|\right) \mathrm{d} s-\int_{-M}^{M} \int_{\gamma_{n}} \frac{\kappa_{n}}{\left|\kappa_{n}\right|}\left(g_{n}\right)_{s s} \mathrm{~d} s \mathrm{~d} u .
\end{aligned}
$$

Letting $n \rightarrow+\infty$ along the subsequence on which $\gamma_{n} \rightarrow \gamma_{\infty}$, we get

$$
\int_{\gamma}\left|\kappa\left(x, t_{n}+\frac{M}{k_{n}^{2}}\right)\right| \mathrm{d} s-\int_{\gamma}\left|\kappa\left(x, t_{n}-\frac{M}{k_{n}^{2}}\right)\right| \mathrm{d} s \rightarrow 0 .
$$

We argue as in (31), using the definition of $g_{n}$ and the fact that, by (19), $L_{t}\left(\gamma_{n}\right) \leqslant k_{n} K$ for some constant $K$ just depending on $\|g\|_{\infty}$ and $T$,

$$
\left|\int_{-M}^{M} \int_{\gamma_{n}} \frac{\kappa_{n}}{\left|\kappa_{n}\right|}\left(g_{n}\right)_{s s} \mathrm{~d} s \mathrm{~d} u\right| \leqslant \int_{-M}^{M} \frac{\left\|\nabla^{2} g\right\|_{\infty}}{k_{n}^{3}} L_{u}\left(\gamma_{n}\right)+\frac{\|\nabla g\|_{\infty}}{k_{n}^{2}}\left(\int_{\gamma_{n}}\left|\kappa_{n}\right| \mathrm{d} s\right) \mathrm{d} u \leqslant \frac{C}{k_{n}^{2}} \rightarrow 0
$$

as $n \rightarrow+\infty$. In particular, this gives

$$
-2 \int_{-M}^{M} \sum_{x, \kappa_{n}(x, u)=0}\left|\left(\kappa_{n}\right)_{s}\right| d u \rightarrow 0 \quad \text { as } n \rightarrow+\infty,
$$

and we can conclude as in [2, Theorem 7.7] that $\gamma_{\infty}$ is a convex eternal solution to the curvature flow, that is, $\gamma_{\infty}$ is the so-called Grim Reaper. 


\subsection{The embedded case}

In this section we strengthen Proposition 2.8 in the case of embedded planar curves.

Following [10] we define

$$
\eta(t):=\inf _{x<y} \frac{|\gamma(x, t)-\gamma(y, t)|}{L_{x, y}(t)}
$$

where

$$
L_{x, y}(t):=\min \left(\int_{x}^{y}\left|\gamma_{x}(\sigma, t)\right| \mathrm{d} \sigma, \int_{0}^{x}\left|\gamma_{x}(\sigma, t)\right| \mathrm{d} \sigma+\int_{y}^{1}\left|\gamma_{x}(\sigma, t)\right| \mathrm{d} \sigma\right) .
$$

Notice that the infimum in (34) is in fact a minimum, moreover $\eta$ is a continuous function in $[0, T)$, where $T$ is the first singularity time. Since the initial curve is embedded, we also have $\eta(0)>0$. Let now

$$
\mathcal{E}(t):=\left\{(x, y): x<y \text { and } \eta(t)=\frac{|\gamma(x, t)-\gamma(y, t)|}{L_{x, y}(t)}\right\} .
$$

In the following we assume for simplicity that $L_{x, y}(t)=\int_{x}^{y}\left|\gamma_{x}(\sigma, t)\right| d \sigma$, the other case being analogous. Notice that, if $\eta(t)<\sqrt{2} / 2$, we have the estimate

$$
\int_{x}^{y}|\kappa|\left|\gamma_{x}(\sigma, t)\right| \mathrm{d} \sigma \geqslant \max _{z, w \in[x, y]}|\tau(z, t)-\tau(w, t)|>\frac{\pi}{2}
$$

for all $(x, y) \in \mathcal{E}(t)$. Indeed, if $|\tau(z, t)-\tau(w, t)| \leqslant \pi / 2$ for all $z, w \in[x, y]$, then $\gamma([x, y])$ is the graph of a 1-Lipschitz function, which in turn implies $\eta(t) \geqslant \sqrt{2} / 2$. Letting $c:=\pi / 2$, from (35) we get

$$
\begin{aligned}
\int_{x}^{y} \kappa(\kappa+g)\left|\gamma_{x}(\sigma, t)\right| \mathrm{d} \sigma & \geqslant \frac{1}{L_{x, y}}\left(\int_{x}^{y}|\kappa|\left|\gamma_{x}(\sigma, t)\right| \mathrm{d} \sigma\right)^{2}-\|g\|_{\infty} \int_{x}^{y}|\kappa|\left|\gamma_{x}(\sigma, t)\right| \mathrm{d} \sigma \\
& \geqslant c\left(\frac{c}{L_{x, y}}-\|g\|_{\infty}\right) \geqslant 0
\end{aligned}
$$

whenever $L_{x, y} \leqslant c /\|g\|_{\infty}$. Moreover, reasoning as in [10], from the minimality condition it follows that

$$
(\kappa(x) \nu(x)-\kappa(y) \nu(y)) \cdot(x-y) \geqslant 0
$$

for all $(x, y) \in \mathcal{E}(t)$. When $\eta(t)<\sqrt{2} / 2$, using (36), (37) and the so called Hamilton's trick (see [15]) we compute

$$
\begin{aligned}
\dot{\eta}(t) & =\min _{(x, y) \in \mathcal{E}(t)} \frac{1}{L_{x, y}}\left[[(\kappa(x)+g(x)) v(x)-(\kappa(y)+g(y)) \nu(y)] \cdot \frac{x-y}{|x-y|}+\eta \int_{x}^{y} \kappa(\kappa+g)\left|\gamma_{x}\right| \mathrm{d} \sigma\right] \\
& \geqslant \min _{(x, y) \in \mathcal{E}(t)}\left[-\frac{2\|g\|_{\infty}}{L_{x, y}}+\frac{c \eta}{L_{x, y}}\left(\frac{c}{L_{x, y}}-\|g\|_{\infty}\right)\right] \\
& \geqslant \min _{(x, y) \in \mathcal{E}(t)}\left[-\left(2+\frac{\pi \sqrt{2}}{4}\right) \frac{\|g\|_{\infty}}{L_{x, y}}+\frac{\pi^{2}}{4 L_{x, y}^{2}} \eta\right] .
\end{aligned}
$$


THEOREM 2.15 Let $\gamma_{0}$ be an embedding and let $T$ be the maximal existence time of (4). Then

$$
T \geqslant c\left(\gamma_{0},\|g\|_{\infty}\right)
$$

Proof. Remark 2.13 ensures that the statement is true if the evolution develops a type I singularity at $t=T$. Now, we can assume that the evolution develops a type II singularity at $t=T$. In particular it follows that $\eta(T)=0$. Let $\tau:=\sup \{t \in[0, T]: \eta(s)>0$ on $[0, t]\}$. Notice that $\tau>0$ due to the fact that $\gamma_{0}$ is an embedding.

The conclusion will follow if we show that $\tau$ is bounded below by a constant depending only on $\gamma_{0}$ and $\|g\|_{\infty}$. Since $\eta(\tau)=0$, we can find $0 \leqslant t_{1}<t_{2} \leqslant \tau$ such that

$$
\eta\left(t_{1}\right)=\bar{\eta}:=\min (\eta(0), \sqrt{2} / 2), \quad \eta\left(t_{2}\right)=\bar{\eta} / 2, \quad \eta(t) \in(\bar{\eta}, \bar{\eta} / 2) \quad \text { for all } t \in\left(t_{1}, t_{2}\right) .
$$

In particular, letting $a:=(2+\pi \sqrt{2} / 4)\|g\|_{\infty}$ and $b:=\pi^{2} / 4$, from (38) we have

$$
\dot{\eta} \geqslant-\frac{a}{L_{x, y}}+\frac{b}{L_{x, y}^{2}} \eta \geqslant-\frac{a}{L_{x, y}}+\frac{b \bar{\eta}}{2 L_{x, y}^{2}} \geqslant-\frac{a^{2}}{2 b \bar{\eta}},
$$

which implies

$$
\tau \geqslant t_{2}-t_{1} \geqslant \frac{b \bar{\eta}^{2}}{a^{2}}=\frac{2 \bar{\eta}^{2}}{(1+4 \sqrt{2} / \pi)^{2}\|g\|_{\infty}^{2}} .
$$

\subsection{The graph case}

We assume now that the curve can be parametrized as $\gamma(x, t)=(x, u(x, t)), x \in[0,1]$, with the following periodic-type boundary conditions:

$$
\begin{aligned}
u(0, t)-u(0,0) & =u(1, t)-u(1,0), \\
u_{x}(0, t) & =u_{x}(1, t),
\end{aligned}
$$

for all $t \in[0, T]$. Notice that $\gamma$ is not a closed curve, but it can be extended to a periodic infinite curve, that is, it is a closed curve on a suitable flat torus, and all the results of the previous sections also apply to this case. In this parametrization, equation (4) becomes

$$
u_{t}=\frac{u_{x x}}{1+u_{x}^{2}}+g(x, u(x)) \sqrt{1+u_{x}^{2}} .
$$

We say that $\gamma$ solves (41) if $\gamma(x, t)=(x, u(x, t))$, where the function $u$ solves (41).

Let us recall the following interpolation inequalities [16].

Proposition 2.16 Let $u \in H^{1}([0,1]) \cap L^{p}([0,1])$, with $p \in[2,+\infty]$. We have

$$
\|u\|_{L^{p}} \leqslant C_{p}\left\|u_{x}\right\|_{L^{2}}^{(p-2) / 2 p}\|u\|_{L^{2}}^{(p+2) / 2 p}+B_{p}\|u\|_{L^{2}}
$$

where the constants $C_{p}, B_{p}$ depend only on $p$. 
The following result can be easily derived from Proposition 2.16 (see [3]). We recall that $L^{p}(\gamma)$ is the intrinsic $L^{p}$ space on the curve $\gamma$ [3].

Proposition 2.17 Let $z$ be a smooth function defined on the support of $\gamma$, where $\gamma$ is a $\mathcal{C}^{1}$ curve, and let $p \in[2,+\infty]$. We have

$$
\|z\|_{L^{p}(\gamma)} \leqslant C_{p}\left\|z_{s}\right\|_{L^{2}(\gamma)}^{(p-2) / 2 p}\|z\|_{L^{2}(\gamma)}^{(p+2) / 2 p}+B_{p}\|z\|_{L^{2}(\gamma)},
$$

where the constants $C_{p}, B_{p}$ depend on $p$ but are independent of $\gamma$.

In particular, for $p=4$, (43) becomes

$$
\int_{\gamma} z^{4} \mathrm{~d} s=\|z\|_{L^{4}(\gamma)}^{4} \leqslant C\left(\left\|z_{s}\right\|_{L^{2}(\gamma)}\|z\|_{L^{2}(\gamma)}^{3}+\|z\|_{L^{2}(\gamma)}^{4}\right) .
$$

LEMMA 2.18 Let $u$ be a smooth solution of (40)-(41), and let

$$
F(x):=\int_{0}^{x} \arctan (t) \mathrm{d} t=x \arctan (x)-\log \sqrt{1+x^{2}}
$$

We have

$$
\begin{gathered}
\partial_{t} \int_{0}^{1} \sqrt{1+u_{x}^{2}} \mathrm{~d} x \leqslant C \int_{0}^{1} \sqrt{1+u_{x}^{2}} \mathrm{~d} x, \\
\partial_{t} \int_{0}^{1} F\left(u_{x}\right) \mathrm{d} x \leqslant C \int_{0}^{1}\left(1+u_{x}^{2}\right) \mathrm{d} x, \\
\partial_{t} \int_{0}^{1}\left(\sqrt{1+u_{x}^{2}}\right)^{3} \mathrm{~d} x \leqslant C+C\left(\int_{0}^{1}\left(\sqrt{1+u_{x}^{2}}\right)^{3} \mathrm{~d} x\right)^{3},
\end{gathered}
$$

where the constants $C>0$ depend only on $\|g\|_{L^{\infty}}$.

Proof. Inequality (45) can be obtained exactly as (20).

In order to show (46), we compute

$$
\begin{aligned}
\partial_{t} \int_{0}^{1} F\left(u_{x}\right) \mathrm{d} x & =\int_{0}^{1}-u_{t}\left(\arctan u_{x}\right)_{x} \mathrm{~d} x=\int_{0}^{1}\left(-u_{t}^{2}+g u_{t} \sqrt{1+u_{x}^{2}}\right) \mathrm{d} x \\
& \leqslant \int_{0}^{1} \frac{g^{2}}{4}\left(1+u_{x}^{2}\right) \mathrm{d} x
\end{aligned}
$$

which leads to (46).

We now prove (47). Letting $e_{1}=(1,0) \in \mathbb{R}^{2}$ and $z:=1 /\left(\tau \cdot e_{1}\right)=\sqrt{1+u_{x}^{2}}$, from (7) we get

$$
z_{t}=-(\kappa+g)_{s} z^{2} v \cdot e_{1}
$$


We compute

$$
\begin{aligned}
\partial_{t} \int_{\gamma} z^{2} \mathrm{~d} s & =\int_{\gamma}\left(2 z z_{t}-\kappa(\kappa+g) z^{2}\right) \mathrm{d} s=\int_{\gamma}\left(-2 z^{3}(\kappa+g)_{s} v \cdot e_{1}-\kappa(\kappa+g) z^{2}\right) \mathrm{d} s \\
& =\int_{\gamma}(\kappa+g)\left(-3 \kappa z^{2}+6 z^{2} z_{s} v \cdot e_{1}\right) \mathrm{d} s=3 \int_{\gamma}(\kappa+g) z_{s} \frac{1+2 z^{2}\left(v \cdot e_{1}\right)^{2}}{v \cdot e_{1}} \mathrm{~d} s \\
& =3 \int_{\gamma}\left(-\frac{2 z^{2}-1}{z^{2}-1} z_{s}^{2}+g z_{s} \frac{2 z^{2}-1}{v \cdot e_{1}}\right) \mathrm{d} s \\
& \leqslant 3 \int_{\gamma}\left(-\frac{2 z^{2}-1}{z^{2}-1} z_{s}^{2}+\left(g z \sqrt{2 z^{2}-1}\right)\left(\sqrt{\frac{2 z^{2}-1}{z^{2}-1}} z_{s}\right)\right) \mathrm{d} s \\
& \leqslant 3 \int_{\gamma}\left(-z_{s}^{2}+\frac{\|g\|_{\infty}^{2}}{2} z^{2}\left(2 z^{2}-1\right)\right) \mathrm{d} s \leqslant 3 \int_{\gamma}\left(-z_{s}^{2}+\|g\|_{\infty}^{2} z^{4}\right) \mathrm{d} s \\
& \leqslant-3\left\|z_{s}\right\|_{L^{2}(\gamma)}^{2}+C\|g\|_{\infty}^{2}\left(\left\|z_{s}\right\|_{L^{2}(\gamma)}\|z\|_{L^{2}(\gamma)}^{3}+\|z\|_{L^{2}(\gamma)}^{4}\right) \\
& \leqslant C\|g\|_{\infty}^{4}\left(\int_{\gamma} z^{2} \mathrm{~d} s\right)^{3}+C\|g\|_{\infty}^{2}\left(\int_{\gamma} z^{2} \mathrm{~d} s\right)^{2}
\end{aligned}
$$

where we used (44) to estimate $\|z\|_{L^{4}}$.

Proposition 2.19 Let $g \in C^{\infty}\left([0,1]^{2}\right)$, and let $u_{0} \in C^{\infty}([0,1])$, with $u_{0 x}(0)=u_{0 x}(1)$. Then there exists $T>0$ depending only on $\left\|u_{0}\right\|_{W^{1, \infty}}$ and $\|g\|_{L^{\infty}}$ such that equations (40)-(41) admit a smooth solution $u \in C^{\infty}([0,1] \times[0, T])$.

Moreover $\|u(t, \cdot)\|_{H^{1}([0,1])} \leqslant K$ for every $t \in[0, T]$, where $K$ depends only on $\left\|u_{0}\right\|_{W^{1, \infty}}$ and $\|g\|_{L^{\infty}}$.

Proof. By standard parabolic regularity theory [14], it is enough to show that the gradient $u_{x}$ remains bounded for a time $T$ as above. From (47) we deduce that there exists $T_{1}>0$, depending only on $\left\|u_{0}\right\|_{W^{1, \infty}}$ and $\|g\|_{L^{\infty}}$, such that $u_{x}(\cdot, t) \in L^{3}([0,1])$ for all $t \in\left[0, T_{1}\right]$. Moreover, by Theorem 2.15 we also have $\kappa=u_{x x}\left(1+u_{x}^{2}\right)^{-3 / 2} \in L^{\infty}\left([0,1] \times\left[0, T_{2}\right]\right)$ for some $T_{2}>0$ depending only on $\left\|u_{0}\right\|_{W^{1, \infty}}$ and $\|g\|_{L^{\infty}}$. As a consequence we get $u_{x} \in L^{\infty}([0,1] \times[0, T])$ with $T=\min \left(T_{1}, T_{2}\right)$.

LEMMA 2.20 We have a continuous embedding

$$
H^{1}\left([0, T], L^{2}([0,1])\right) \cap L^{\infty}\left([0, T], H^{1}([0,1])\right) \hookrightarrow C^{1 / 2,1 / 4}([0,1] \times[0, T]) .
$$

Proof. Let $u \in H^{1}\left([0, T], L^{2}([0,1])\right) \cap L^{\infty}\left([0, T], H^{1}([0,1])\right)$, and let $(x, t),(y, s) \in[0,1] \times$ $[0, T]$, with $x<y, t<s$. Since $u \in L^{\infty}\left([0, T], H^{1}([0,1])\right)$, we have

$$
|u(x, t)-u(y, t)| \leqslant \int_{x}^{y}\left|u_{x}\right| \mathrm{d} \sigma \leqslant C \sqrt{x-y} .
$$

Moreover, since also $u \in H^{1}\left([0, T], L^{2}([0,1])\right)$, we have

$$
\|u(\cdot, t)-u(\cdot, s)\|_{L^{2}}^{2}=\int_{0}^{1}|u(x, t)-u(x, s)|^{2} \mathrm{~d} x \leqslant(s-t) \int_{[0,1] \times[0, T]} u_{t}^{2} \mathrm{~d} x \mathrm{~d} t \leqslant C(s-t) .
$$


By (42) with $p=\infty$, this implies

$$
\begin{aligned}
\|u(\cdot, t)-u(\cdot, s)\|_{L^{\infty}} & \leqslant C\left(\left\|u_{x}(\cdot, t)-u_{x}(\cdot, s)\right\|_{L^{2}}^{1 / 2}\|u(\cdot, t)-u(\cdot, s)\|_{L^{2}}^{1 / 2}+\|u(\cdot, t)-u(\cdot, s)\|_{L^{2}}\right) \\
& \leqslant C(s-t)^{1 / 4}
\end{aligned}
$$

The conclusion follows from (50) and (51).

Proposition 2.21 Let $u_{0} \in W^{1, \infty}([0,1])$, let $g_{n} \in C^{\infty}\left([0,1]^{2}\right) \cap L^{\infty}\left([0,1]^{2}\right)$ with $\left\|g_{n}\right\|_{L^{\infty}} \leqslant C$ for every $n$, and let $u_{0 n} \in C^{\infty}([0,1])$ be such that $\left\|u_{0 n}\right\|_{W^{1, \infty}} \leqslant L$ for every $n$ and $u_{0_{n}}$ converges to $u_{0}$ uniformly on $[0,1]$. Then, letting $u_{n} \in C^{\infty}([0,1] \times[0, T])$ be the solutions of (40)-(41) given by Proposition 2.19, with $g=g_{n}$ and with initial data $u_{0 n}$, there exists $u \in H^{1}\left([0, T], L^{2}([0,1])\right) \cap$ $L^{\infty}\left([0, T], H^{1}([0,1])\right)$ such that, up to a subsequence, $u_{n} \rightarrow u$ uniformly on $[0,1] \times[0, T]$.

Proof. By Proposition 2.19 there exist $T>0$, depending only on $C$ and $L$, such that the solutions $u_{n}$ are uniformly bounded in $L^{\infty}\left([0, T], H^{1}([0,1])\right)$. Moreover, using the equality for (48), we obtain

$$
\int_{0}^{1} \frac{\left(u_{n}\right)_{t}^{2}}{2} \mathrm{~d} x \leqslant \frac{\left\|g_{n}\right\|_{\infty}^{2}}{2} \int_{0}^{1}\left(1+u_{n_{x}}^{2}\right) \mathrm{d} x-\partial_{t} \int_{0}^{1} F\left(\left(u_{n}\right)_{x}\right) \mathrm{d} x
$$

and integrating it in time we also get a uniform bound of $u_{n}$ in $H^{1}\left([0, T], L^{2}([0,1])\right)$. It then follows that the sequence $u_{n}$ converges, up to a subsequence as $n \rightarrow+\infty$, to a limit function $u$ in the weak topology of $H^{1}\left([0, T], L^{2}([0,1])\right) \cap L^{\infty}\left([0, T], H^{1}([0,1])\right)$. By Lemma $2.20, u_{n}$ are uniformly Hölder continuous and then we conclude by the Arzelà-Ascoli theorem that, along a subsequence, $u_{n} \rightarrow u$ uniformly on $[0,1] \times[0, T]$.

We are interested in studying solutions of (41) when $g$ is only an $L^{\infty}$-function. We consider the simpler case in which $g$ is independent of $u$, i.e. $g(x, y)=g(x)$. In this case we define the following notion of weak solution.

Definition 2.22 We say that a function $u \in H^{1}\left([0, T], L^{2}([0,1])\right) \cap L^{\infty}\left([0, T], H^{1}([0,1])\right)$ is a weak solution of (41) if

$$
\int_{[0,1] \times[0, T]}\left(u_{t} \varphi+\arctan \left(u_{x}\right) \varphi_{x}-g(x) \sqrt{1+u_{x}^{2}} \varphi\right) \mathrm{d} x \mathrm{~d} t=0
$$

for all test functions $\varphi \in C_{c}^{1}([0,1] \times(0, T))$, with periodic boundary conditions.

We have the following existence theorem for weak solutions to (41).

TheOREM 2.23 Let $g(x, y)=g(x)$, with $g \in L^{\infty}([0,1])$, and let $u_{0} \in W^{2, \infty}([0,1])$ satisfy $u_{0 x}(0)=u_{0 x}(1)$. Then there exists $T>0$ depending only on $u_{0}$ and $\|g\|_{\infty}$ such that equation (41) admits a weak solution $u \in W^{1, \infty}\left([0, T], L^{\infty}([0,1])\right) \cap L^{\infty}\left([0, T], W^{2, \infty}([0,1])\right)$ with initial datum $u_{0}$.

Proof. Let $g_{n} \in C^{\infty}([0,1])$ be a sequence of smooth functions which converge to $g$ weakly* in $L^{\infty}([0,1])$. By Propositions 2.19 and 2.21 there exist $T>0$, depending only on $\left\|u_{0}\right\|_{H^{1}}$ and $\|g\|_{L^{\infty}}$, and smooth solutions $u_{n}$ of (41) which converge, up to a subsequence, to a limit function $u$ uniformly and in the weak topology of $H^{1}\left([0, T], L^{2}([0,1])\right) \cap L^{\infty}\left([0, T], H^{1}([0,1])\right)$. 
Let us prove that $u$ is a weak solution of (41). The main point is showing that $u_{n x}$ converges to $u_{x}$ almost everywhere, so that we can pass to the limit in (52). We compute

$$
\begin{aligned}
\partial_{t} \frac{u_{t}^{2}}{2} & =u_{t} u_{t t}=u_{t}\left(\frac{u_{x x}}{1+u_{x}^{2}}+g(x) \sqrt{1+u_{x}^{2}}\right)_{t} \\
& =\frac{u_{t} u_{t x x}}{1+u_{x}^{2}}-2 \frac{u_{x} u_{x x}}{\left(1+u_{x}^{2}\right)^{2}}\left(\frac{u_{t}^{2}}{2}\right)_{x}+g \frac{u_{x}}{\sqrt{1+u_{x}^{2}}}\left(\frac{u_{t}^{2}}{2}\right)_{x} \\
& \leqslant \frac{1}{1+u_{x}^{2}}\left(\frac{u_{t}^{2}}{2}\right)_{x x}+\left(g \frac{u_{x}}{\sqrt{1+u_{x}^{2}}}-2 \frac{u_{x} u_{x x}}{\left(1+u_{x}^{2}\right)^{2}}\right)\left(\frac{u_{t}^{2}}{2}\right)_{x} .
\end{aligned}
$$

In particular, applying the same computation as in (53) to $u_{n}$, we find that $\left\|u_{n t}\right\|_{\infty}$ is decreasing in time. Indeed if $M_{n}(t)=\sup _{x \in[0,1]} u_{n t}^{2} / 2$, (53) gives that $M_{n}^{\prime}(t) \leqslant 0$. Therefore $u \in$ $W^{1, \infty}\left([0, T], L^{\infty}([0,1])\right)$. Moreover, since $g$ depends only on $x$ we have

$$
\begin{aligned}
\partial_{t} \int_{0}^{1} \frac{u_{t}^{2}}{2} \mathrm{~d} x & =\int_{0}^{1} u_{t} u_{t t} \mathrm{~d} x=\int_{0}^{1}-\arctan \left(u_{x}\right)_{t} u_{x t}+g\left(\sqrt{1+u_{x}^{2}}\right)_{t} u_{t} \mathrm{~d} x \\
& \leqslant \int_{0}^{1}\left(-\frac{u_{x t}^{2}}{1+u_{x}^{2}}+g u_{x} u_{t} \frac{u_{x t}}{\sqrt{1+u_{x}^{2}}}\right) \mathrm{d} x \\
& \leqslant \int_{0}^{1}\left(-\frac{u_{x t}^{2}}{1+u_{x}^{2}}+\frac{g^{2} u_{x}^{2} u_{t}^{2}}{2}\right) \mathrm{d} x \leqslant \frac{1}{2}\|g\|_{\infty}^{2}\left\|u_{t}\right\|_{\infty}^{2}\left\|u_{x}\right\|_{L^{2}}^{2}=C
\end{aligned}
$$

where the constant $C>0$ depends only on $u_{0}$ and $\|g\|_{\infty}$. We then get

$$
\begin{aligned}
\int_{[0,1]}\left(\arctan \left(u_{x}\right)\right)_{x}^{2} \mathrm{~d} x & =\int_{[0,1]}\left(u_{t}-g \sqrt{1+u_{x}^{2}}\right)^{2} \mathrm{~d} x \leqslant C \quad \forall t \in[0, T] \\
\int_{[0,1] \times[0, T]}\left(\arctan \left(u_{x}\right)\right)_{t}^{2} \mathrm{~d} x \mathrm{~d} t & =\int_{[0,1] \times[0, T]} \frac{u_{x t}^{2}}{1+u_{x}^{2}} \mathrm{~d} x \mathrm{~d} t \leqslant C .
\end{aligned}
$$

As a consequence, the function $\arctan \left(u_{n x}\right)$ is uniformly bounded in $H^{1}\left([0, T], L^{2}([0, T])\right) \cap$ $L^{\infty}\left([0, T], H^{1}([0,1])\right)$. Therefore, the sequence $\arctan \left(u_{n x}\right)$ converges, up to a subsequence, to $\arctan \left(u_{x}\right)$ uniformly on $[0,1] \times[0, T]$. Since $\arctan$ is injective this implies that the sequence $u_{n x}$ converges to $u_{x}$ a.e. on $[0,1] \times[0, T]$, and we can pass to the limit in (52), concluding that $u$ is a weak solution of (41).

Finally, as $\arctan \left(u_{x}\right)$ is continuous, possibly reducing $T$ we see that $u_{x}$ is also continuous (hence bounded) on $[0,1] \times[0, T]$. In particular, recalling (41) the uniform bound on $u_{t}$ implies an analogous bound on $u_{x x}$, that is, $u \in L^{\infty}\left([0, T], W^{2, \infty}([0,1])\right)$.

REMARK 2.24 If $u_{0}$ is only in $H^{1}([0,1])$, since the sequence $u_{n}$ is uniformly bounded in $H^{1}\left([0, T], L^{2}([0, T])\right)$, reasoning as in Theorem 2.23 we get $u \in W_{\text {loc }}^{1, \infty}\left((0, T], L^{\infty}([0,1])\right) \cap$ $L_{\mathrm{loc}}^{\infty}\left((0, T], W^{2, \infty}([0,1])\right)$.

We conclude the section with a comparison and uniqueness result for solutions to (41).

THEOREM 2.25 Let $g(x, y)=g(x)$, with $g \in L^{\infty}([0,1])$, and let $u_{1}$, $u_{2}$ be two solutions to (41) such that $u_{1}(x, 0) \leqslant u_{2}(x, 0)$ for all $x \in[0,1]$. Then

$$
u_{1} \leqslant u_{2} \quad \text { on }[0,1] \times[0, T] .
$$

In particular, there is a unique solution to (41), given an initial datum $u_{0} \in W^{2, \infty}([0,1])$. 
Proof. Let

$$
d(t):=\min _{x \in[0,1]}\left(u_{2}(x, t)-u_{1}(x, t)\right) .
$$

Possibly replacing $u_{1}(\cdot, 0)$ with $u_{1}(\cdot, 0)-\delta$, we can assume that $d(0)=\delta>0$. The assertion now follows if we can show that $d(t) \geqslant \delta$ for all $t \in[0, T]$. Let $w=u_{2}-u_{1}$, so that

$$
w_{t}=\frac{\left(u_{2}\right)_{x x}}{1+\left(u_{2}\right)_{x}^{2}}-\frac{\left(u_{1}\right)_{x x}}{1+\left(u_{1}\right)_{x}^{2}}+g(x)\left(\sqrt{1+\left(u_{2}\right)_{x}^{2}}-\sqrt{1+\left(u_{1}\right)_{x}^{2}}\right) .
$$

From (54) it follows that

$$
w_{t} \in L^{2}\left([0, T], H^{1}([0,1])\right) \hookrightarrow L^{2}\left([0, T], C^{\alpha}([0,1])\right)
$$

for all $\alpha<1 / 2$. Choose now $t \in[0, T]$ such that $w_{t}(\cdot, t) \in C^{\alpha}([0,1])$ and notice that, for all $x \in[0,1]$ such that $d(t)=w(x, t)$, we have

$$
w_{x}=\left(u_{2}\right)_{x}-\left(u_{1}\right)_{x}=0 .
$$

In particular, recalling (55), $w$ is twice differentiable at $x$ and we have

$$
w_{t}=\frac{\left(u_{2}\right)_{x x}}{1+\left(u_{2}\right)_{x}^{2}}-\frac{\left(u_{1}\right)_{x x}}{1+\left(u_{1}\right)_{x}^{2}}=\frac{w_{x x}}{1+\left(u_{1}\right)_{x}^{2}} \geqslant 0 .
$$

For almost every $t \in[0, T]$ we then get

$$
\dot{d}(t)=\min _{x: d(t)=w(x, t)} w_{t}(x, t) \geqslant 0,
$$

which gives the conclusion.

REMARK 2.26 We point out that in general we have $T<+\infty$ in Theorem 2.23, since the derivative $u_{x}$ may blow up in finite time. This is related to the so-called fingering phenomenon, and we shall give an explicit example of such behaviour. Let $u_{0}=0, g(x)=M$ for $x \in[0,1 / 2)$, and $g(x)=-M$ for $x \in[1 / 2,1]$, where the constant $M$ is greater than 4 . For all $t \geqslant 0$, we set

$$
\begin{array}{ll}
u^{-}(x, t):=\sqrt{\frac{x}{2}-x^{2}}-\frac{1}{4}+(M-4) t, & x \in(0,1 / 2), \\
u^{+}(x, t):=\sqrt{\frac{3}{2} x-x^{2}-\frac{1}{2}}+\frac{1}{4}-(M-4) t, & x \in(1 / 2,1) .
\end{array}
$$

A direct computation shows that $u^{-}, u^{+}$are respectively a subsolution and a supersolution of (41) on their intervals of definition. By Theorem 2.25, for all $t \in[0, T]$ it follows that $u(\cdot, t) \geqslant u^{-}(\cdot, t)$ on $(0,1 / 2)$, and $u(\cdot, t) \leqslant u^{+}(\cdot, t)$ on $(1 / 2,1)$. Since $u$ is continuous, this necessarily implies $T \leqslant$ $1 /(4(M-4))$. More precisely, if we extend the solution $u$ on a maximal time interval $\left[0, T_{\max }\right)$, we have $T_{\max } \leqslant 1 /(4(M-4))$ and the derivative $u_{x}(1 / 2, t)$ blows up (in absolute value) as $t \rightarrow T_{\max }$. 


\section{A homogenization problem}

Given a smooth function $g$ which is periodic on $[0,1]^{2}$, we consider the following homogenization problem:

$$
u_{t}=\frac{u_{x x}}{1+u_{x}^{2}}+g\left(\frac{x}{\varepsilon}, \frac{u}{\varepsilon}\right) \sqrt{1+u_{x}^{2}}
$$

with initial data $u(x, 0)=u_{0}(x)$, satisfying (40).

Notice that, after the parabolic rescaling $s=t / \varepsilon^{2}, y=x / \varepsilon$ and $v=u / \varepsilon$, problem (56) becomes

$$
v_{s}=\frac{v_{y y}}{1+v_{y}^{2}}+\varepsilon g(y, v) \sqrt{1+v_{y}^{2}} .
$$

In [6] existence of traveling wave solutions for (57) has been established. Moreover in [13] (see also [5]) the authors discuss the uniqueness of traveling fronts and characterize the asymptotic speed in some particular case.

A straightforward application of the results in Subsection 2.6 gives the first result about the convergence of the solutions to the perturbed problem (56).

Proposition 3.1 Let $u_{0 \varepsilon} \in C^{\infty}([0,1])$ satisfy (40) and $\left\|u_{0 \varepsilon}\right\|_{W^{1, \infty}} \leqslant L$, and let $u_{\varepsilon}$ be the solution to (56) with initial data $u_{0 \varepsilon}$. Then, up to a subsequence,

$$
u_{\varepsilon} \rightarrow u \in H^{1}\left([0, T], L^{2}([0,1])\right) \cap L^{\infty}\left([0, T], H^{1}([0,1])\right) \quad \text { as } \varepsilon \rightarrow 0,
$$

uniformly on $[0,1] \times[0, T]$.

Proof. By Proposition 2.19 there exists $T>0$ independent of $\varepsilon$ and a family of smooth solutions $u_{\varepsilon}$ of (56), which are uniformly bounded in $H^{1}\left([0, T], L^{2}([0,1])\right) \cap L^{\infty}\left([0, T], H^{1}([0,1])\right)$. In particular, as in Propositon 2.21, we can pass to the limit, up to a subsequence as $\varepsilon \rightarrow 0$, to find that

$$
u_{\varepsilon} \rightarrow u \in H^{1}\left([0, T], L^{2}([0,1])\right) \cap L^{\infty}\left([0, T], H^{1}([0,1])\right) \quad \text { uniformly on }[0,1] \times[0, T] .
$$

There are two main open problems related to the homogenization of equation (56):

1) the characterization of $u$ as the solution of an appropriate homogenized equation;

2) the convergence on large time intervals.

Concerning the second question, we expect the following result:

Conjecture 3.2 The convergence in Proposition 3.1 is uniform on $[0,1] \times[0,+\infty)$.

Let us give a heuristic argument supporting our conjecture. Due to the comparison principle and the periodicity of $g$, for all $N \in \mathbb{N}$ we have the estimate

$$
\left|u_{\varepsilon}(x, t)-u_{\varepsilon}(x+N \varepsilon, t)\right| \leqslant([L]+1) N \varepsilon,
$$

where $[L]$ denotes the integer part of $L$. Passing to the limit in (58) as $\varepsilon \rightarrow 0$, we get

$$
|u(x, t)-u(y, t)| \leqslant L|y-x|,
$$

that is, the norm $\|u(\cdot, t)\|_{W^{1, \infty}}$ is non-increasing in $t$. We expect this bound to be true also for the approximating sequence $u_{\varepsilon}$, which would imply that we can take $T=+\infty$.

Concerning the first question, we have only some partial results. We state a result when $g$ depends only on $x$. 
THEOREM 3.3 Let $g(x, y)=g(x) \in L^{\infty}([0,1])$, and let $u_{0} \in W^{2, \infty}([0,1])$ with $u_{0 x}(0)=$ $u_{0 x}(1)$. Then there exists $T>0$ depending only on $u_{0}$ and $\|g\|_{\infty}$ such that the solutions $u_{\varepsilon}$ to (56) converge in $W^{1, \infty}\left([0, T], L^{\infty}([0,1])\right) \cap L^{\infty}\left([0, T], W^{2, \infty}([0,1])\right)$, as $\varepsilon \rightarrow 0$, to the unique solution $u$ of

$$
u_{t}=\frac{u_{x x}}{1+u_{x}^{2}}+\left(\int_{0}^{1} g(x) \mathrm{d} x\right) \sqrt{1+u_{x}^{2}}
$$

with initial datum $u_{0}$ and boundary conditions (40). In particular, $u \in C^{\infty}([0,1] \times(0, T])$.

Proof. The proof is a straightforward adaptation of the proof of Theorem 2.23.

In the general case, we can determine the limit equation satisfied by $u$ only in a very specific case, that is, when the initial data are plane-like.

Proposition 3.4 Let $u_{0 \varepsilon} \in C^{\infty}([0,1])$ satisfy (40), $\left\|u_{0 \varepsilon}\right\|_{W^{1, \infty}} \leqslant L$ and $u_{0 \varepsilon}(x) \rightarrow \alpha x$ uniformly in $[0,1]$. Then, if $u_{\varepsilon}$ are the solutions to (56), (40) with initial datum $u_{0 \varepsilon}$, we have

$$
\lim _{\varepsilon \rightarrow 0} u_{\varepsilon}(x, t)=u(x, t):=\alpha x+c(\alpha) \sqrt{1+\alpha^{2}} t,
$$

uniformly in $[0,1] \times[0, T]$, with

$$
c(\alpha):= \begin{cases}0 & \text { if } G(s)=0 \text { for some } s \in[0,1], \\ \left(\int_{0}^{1} \frac{1}{G(s)} \mathrm{d} s\right)^{-1} & \text { otherwise }\end{cases}
$$

where

$$
G(s):=\lim _{L \rightarrow \infty} \frac{1}{L} \int_{0}^{L} g(x, \alpha x+s) \mathrm{d} x .
$$

Proof. By Proposition 2.19 for every $\varepsilon$ there exists a smooth solution to (56) in $[0, T]$, for $T$ independent of $\varepsilon$. Moreover, by Proposition 3.1, these solutions $u_{\varepsilon}$ converge uniformly on $[0,1] \times$ $[0, T]$, up to subsequences, to a function $u$.

By [6, Thm. 4.1] (see also [13]) for all $\alpha \in \mathbb{R}$ there exist global smooth solutions $\hat{u}_{\alpha, \varepsilon}$ of (56), with average slope $\alpha$, which are pulsating waves, that is, there exist $\tau>0$ and a vector $\left(v_{1}, v_{2}\right) \in \mathbb{Z}^{2}$, depending on $(\alpha, \varepsilon)$ and such that

$$
\hat{u}_{\alpha, \varepsilon}(x, t+\tau)=\hat{u}_{\alpha, \varepsilon}\left(x-\varepsilon v_{1}, t\right)+\varepsilon v_{2} \quad \forall(x, t) \in \mathbb{R}^{2} .
$$

We let

$$
c(\alpha, \varepsilon)=\frac{\varepsilon\left(v_{1}, v_{2}\right) \cdot v_{\alpha}}{\tau} \quad \text { where } \quad v_{\alpha}=\left(-\frac{\alpha}{\sqrt{1+\alpha^{2}}}, \frac{1}{\sqrt{1+\alpha^{2}}}\right)
$$

be the velocity of the wave in the normal direction $v_{\alpha}$ and we set $c(\alpha, \varepsilon)=0$ if $\hat{u}_{\alpha, \varepsilon}$ is a standing wave. In particular, in [6, Thm. 4.1] it is shown that $\hat{u}_{\varepsilon}$ can be represented as

$$
\hat{u}_{\alpha, \varepsilon}(x, t)=\alpha x+c(\alpha, \varepsilon) \sqrt{1+\alpha^{2}} t+\mathcal{O}(\varepsilon) \quad \forall(x, t) \in \mathbb{R}^{2},
$$

where $|\mathcal{O}(\varepsilon)| \leqslant C \varepsilon$, for a constant $C$ depending only on (the $C^{2}$-norm of) $g$. Moreover, by [6, Cor. 2.5] the derivatives $\left(\hat{u}_{\alpha, \varepsilon}\right)_{x}(x, t)$ are uniformly bounded for all $(x, t) \in \mathbb{R}^{2}$ and for all $\varepsilon$ small enough. 
Notice that, by [6, Prop. 4.4], for all $(x, t) \in \mathbb{R}^{2}$ we have

$$
\begin{aligned}
& c(\alpha, \varepsilon)=0 \Rightarrow\left(\hat{u}_{\alpha, \varepsilon}\right)_{t}=0, \\
& c(\alpha, \varepsilon)>0 \Rightarrow\left(\hat{u}_{\alpha, \varepsilon}\right)_{t}>0, \\
& c(\alpha, \varepsilon)<0 \Rightarrow\left(\hat{u}_{\alpha, \varepsilon}\right)_{t}<0 .
\end{aligned}
$$

In particular, without loss of generality we can assume that

$$
\left(\hat{u}_{\alpha, \varepsilon}\right)_{t} \geqslant 0 \quad \forall(x, t) \in \mathbb{R}^{2} .
$$

One can now argue as in [5, (4.9) and (4.10)] and conclude by the maximum principle that

$$
\left|\left(\hat{u}_{\alpha, \varepsilon}\right)_{x}-\alpha\right| \leqslant C \varepsilon \quad \forall(x, t) \in \mathbb{R}^{2},
$$

for a constant $C$ depends only on $g$. Integrating (56) on $[0,1]$ and using (61), a direct computation as in [5, Prop. 6] gives $\lim _{\varepsilon \rightarrow 0} c(\alpha, \varepsilon)=c(\alpha)$.

Finally, by the comparison principle for solutions to (56), we can use the functions $\hat{u}_{\alpha, \varepsilon}$ as barriers for $u_{\varepsilon}$, and obtain

$$
\lim _{\varepsilon \rightarrow 0} u_{\varepsilon}(x, t)=u(x, t)=\alpha x+c(\alpha) \sqrt{1+\alpha^{2}} t,
$$

uniformly in $[0,1] \times[0, T]$.

REMARK 3.5 If we assume that $u_{0 \varepsilon}(x) \rightarrow \alpha x$ in $C^{1}([0,1])$, the result of Proposition 3.4 can be strengthened to

$$
\lim _{\varepsilon \rightarrow 0} u_{\varepsilon}(x, t)=u(x, t):=\alpha x+c(\alpha) \sqrt{1+\alpha^{2}} t \quad \text { locally uniformly in }[0,1] \times[0,+\infty) .
$$

Indeed, under this stronger assumption, for every $\varepsilon$ small enough there exists a unique smooth solution $u_{\varepsilon}$ to $(56)$ in $[0,1] \times(0,+\infty)[6$, Thm. 2.7].

REMARK 3.6 Notice that $c(\alpha)=\int_{[0,1]^{2}} g$ for all $\alpha \notin \mathbb{Q}$, so that the function $\alpha \mapsto c(\alpha)$ is not necessarily continuous. This suggests that the homogenization limit of (2) should be a geometric evolution of the form

$$
v=\bar{c}(\nu, \kappa) v
$$

where the function $\bar{c}$ is in general discontinuous.

\section{REFERENCES}

1. Abresch, U., \& Langer, J. The normalized curve shortening flow and homothetic solutions. J. Differential Geom. 23 (1986), 175-196. Z Zbl 0592.53002 MR 0845704

2. Altschuler, S. Singularities of the curve shrinking flow for space curves. J. Differential Geom. 34 (1991), 491-514. Zbl 0754.53006 MR 1131441

3. Aubin, T. Some Nonlinear Problems in Riemannian Geometry. Springer, New York (1998). Zbl 0896.53003 MR 1636569

4. Cardaliaguet, P., Lions, P.-L., \& Souganidis, P. E. A discussion about the homogenization of moving interfaces. J. Math. Pures Appl. 91 (2009), 339-363. Zbl 1180.35070 MR 2518002 
5. Craciun, B., \& BhatTACHARYA, K. Effective motion of a curvature-sensitive interface through a heterogeneous medium. Interfaces Free Bound. 6 (2004), 151-173. Zbl 1061.35148 MR 2079601

6. Dirr, N., Karali, G., \& YIP, N. K. Pulsating wave for mean curvature flow in inhomogeneous medium. Eur. J. Appl. Math. 19 (2008), 661-699. Zbl 1185.53076 MR 2463225

7. ECKER, K., \& HUISKEN, G. Parabolic methods for the construction of spacelike slices of prescribed mean curvature in cosmological spacetimes. Comm. Math. Phys. 135 (1991), 595-613. Zbl 0721.53055 MR 1091580

8. Gage, M., \& Hamilton, R. S. The heat equation shrinking convex plane curves. J. Differential Geom. 23 (1986), 69-96. Z Zbl 0621.53001 MR 0840401

9. Huisken, G. Asymptotic behavior for singularities of the mean curvature flow. J. Differential Geom. 31 (1990), 285-299. Zbl 0694.53005 MR 1030675

10. Huisken, G. A distance comparison principle for evolving curves. Asian J. Math. 2 (1998), 127-133. Zbl 0931.53032 MR 1656553

11. Huisken, G., \& Polden, A. Geometric evolution equations for hypersurfaces. In: Calculus of Variations and Geometric Evolution Problems (Cetraro, 1996), Springer, Berlin (1999), 45-84. Zbl 0942.35047 MR 1731639

12. LiOns, P.-L., \& SougAnidis, P. E. Homogenization of degenerate second-order PDE in periodic and almost periodic environments and applications. Ann. Inst. H. Poincaré Anal. Non Linéaire 22 (2005), 667-677. Zbl 1135.35092 MR 2171996

13. LOU, B., \& CHEN, X. Traveling waves of a curvature flow in almost periodic media. J. Differential Equations 247 (2009), 2189-2208. Zbl 1182.35073 MR 2561275

14. Lunard, A. Analytic Semigroups and Optimal Regularity in Parabolic Problems. Progr. Nonlinear Differential Equations Appl. 16, Birkhäuser, Basel (1995). Z Zbl 0816.35001 MR 1329547

15. MantegazzA, C. Lecture Notes on Mean Curvature Flow. Birkhäuser, Basel (2011). Zbl pre05898720 MR 2815949

16. Nirenberg, L. On elliptic partial differential equations. Ann. Scuola Norm. Sup. Pisa Cl. Sci. 13 (1959), 116-162. Zbl 0088.07601 MR 0109940 